\title{
Coexistence of Superconductivity and Ferromagnetism in Intermetallic Uranium-Based Superconductors UCoGe, UIr and UGe ${ }_{2}$
}

\author{
Haftu Brhane \\ Department of Physics College of Natural and Computational Sciences, Haramaya University, \\ Dire Dawa-P.O.Box138, Ethiopia,
}

\begin{abstract}
Starting with a model Hamiltonian for the system with equal spin singlet and triplet pairings based on quantum field theory and green function formalism, we obtain expressions for superconductivity and ferromagnetism parameters. The model exhibits a distinct possibility of the coexistence of superconductivity and ferromagnetism, which are two usually irreconcilable cooperative phenomena, however, recently ferromagnetism and superconductivity have been shown to coexist simultaneously in the newly discovered compounds such as UCoGe, UIr and $\mathrm{UGe}_{2}$.The work is motivated by the recent experimental evidences below the superconducting phase temperature in a number of uranium-based superconductors. The theoretical results are then applied to show the coexistence of superconductivity and ferromagnetism in the intermetallic uraniumbased compound $\mathrm{UCoGe}$, UIr and $\mathrm{UGe}_{2}$. The limitations of the model are also discussed.
\end{abstract}

Keywords: - BCS Hamiltonian, green function formalism, Order parameters, Spin singlet and triplet state, Superconducting and ferromagnetic

\section{INTRODUCTION}

Since the discovery of superconductivity in itinerant ferromagnet UGe2 under pressure in a limited range $(1 \leq P \leq 1.5 \mathrm{GPa}$ ) below $1 \mathrm{~K}$, a lot of interest has been generated in the study of coexistence of these two cooperative phenomena of superconductivity and ferromagnetism [1] which have been considered until very recently hostile and incompatible.

In fact, ErRh4B4 was the first ferromagnetic superconductor in which superconductivity was found to exist in a small temperature interval with modulated ferromagnetic phase as had been observed in a detailed study by Sinha et al. [2]. The first observation of a zero resistance in the ferromagnetic state of HoMo6S8 was reported by Lynn et al.[3] and then by Genicon et al.[4]. The theoretical work using a model Hamiltonian which takes into account the spin interactions between conduction electrons and ferromagnetically ordered localized electrons, can explain the co-existence of singlet superconductivity and ferromagnetism in HoMo6S8[17].

The intermetallic compound UCoGe belongs to the fascinating family of superconducting ferromagnets $[5,6]$. In superconducting ferromagnets, a superconducting transition takes place at a temperature $T_{\mathrm{sc}}$ deep in the ferromagnetic state, i.e. well below the Curie temperature $T_{\mathrm{C}}$, without expelling magnetic order. The superconducting ferromagnets discovered hitherto are UGe2 (under pressure) [7], URhGe [8], UIr (under pressure) [9], and UCoGe. In these uranium intermetallics magnetism has a strong itinerant character and both ordering phenomena are carried by the same $5 f$ electrons.

The coexistence of superconductivity and ferromagnetism is at odds with the standard BCS theory for phonon-mediated $s$-wave superconductivity, because the ferromagnetic exchange field is expected to inhibit spin-singlet Cooper pairing [10]. Very recently, the discovery of superconductivity in a single crystal of Y9Co7 [11], UGe2 [12], ZrZn2 [13], URhGe [8], UIr [9], and UCoGe [14] revived the interest in the coexistence of superconductivity and ferromagnetism in the same homogenous system.

The coexistence of superconductivity and weak itinerant ferromagnetism in UCoGe was reported in 2007 [5]. Till then UCoGe was thought to be a paramagnet down to a temperature of $1.2 \mathrm{~K}$ [15]. However, in a search for a ferromagnetic quantum critical point induced in the superconducting ferromagnet URhGe $(T \mathrm{~s}=$ $0.25 \mathrm{~K}, T \mathrm{C}=9.5 \mathrm{~K}$ ) by alloying with $\mathrm{Co}[16]$, it was discovered that UCoGe is actually a weak itinerant ferromagnet below $T \mathrm{C}=3 \mathrm{~K}$ and, moreover, a superconductor below $T \mathrm{~s}=0.8 \mathrm{~K}$. 
Neutron scattering experiments confirm that magnetism in UGe2 and URhGe is carried by uranium $f$ electrons. Superconductivity and ferromagnetism coexist in URhGe and UCoGe at ambient pressure. Thermal expansion and specific heat measurement provides solid evidence for bulk ferromagnetism and superconductivity. Proximity to ferromagnetic instability, defect sensitivity of superconducting transition temperature, and absence of Pauli limiting suggest triplet superconductivity mediated by critical ferromagnetic fluctuations although the mechanism of superconductivity coexisting with ferromagnetism is still under debate [18-21].

The standard way to examine a coexistence phase of ferromagnetism and superconductivity is to introduce two kinds of fermions. Ferromagnetism could be caused by local f-electrons whereas superconductivity by itinerant ones. But in these materials such as UCoGe and UIr, both the roles are played by the same uranium $5 \mathrm{f}$ electrons which are itinerant and strongly correlated. Thus, it would be proper to study microscopically a model where the coexistence of both ferromagnetism and superconductivity can be described by only one kind of electrons. Such a model study has recently been initiated by Karchev et al. [22]. However, this model is confined to singlet superconductivity which is unlikely to occur inside a ferromagnet [23].

In this paper, we start with a model Hamiltonian which incorporates not only terms of the BCS but also an additional new term representing interaction between conduction and localized electrons, for the uraniumbased superconductors UCoGe, UIr and $\mathrm{UGe}_{2}$, to examine the coexistence of superconductivity and ferromagnetism

\section{MODEL HAMILTONIAN OF THE SYSTEM}

The purpose of this work is to study theoretically the co-existence of ferromagnetism and superconductivity properties in the compounds UCoGe, UIr and $\mathrm{UGe}_{2}$ in general and to find expression for transition temperature and order parameter in particular. The system under consideration consists of conduction and localized electrons, between which exchange interaction exists. Within the framework of the BCS model, the Hamiltonian of the system can be written as [17]:

where,

$$
H=H_{1}+H_{2}+H_{3}
$$

$$
H_{1}=\sum_{\kappa, \sigma} \epsilon_{\kappa} \hat{a}_{\kappa, \sigma}^{\dagger} \hat{a}_{\kappa, \sigma}+\sum_{l, \sigma} \epsilon_{l} \hat{b}_{l, \sigma}^{\dagger} \hat{b}_{l, \sigma}
$$

represents, the single particle energies of the conduction and the localized electrons, measured relative to the chemical potential. $\hat{a}_{\kappa, \sigma}^{\dagger}\left(\hat{a}_{\kappa, \sigma}\right)$ and $\hat{b}_{l, \sigma}^{\dagger}\left(\hat{b}_{l, \sigma}\right)$ are the creation (annihilation) operators for conduction and localized electrons respectively.

$$
H_{2}=-\sum_{\kappa, \kappa^{\prime}} V_{\kappa \kappa^{\prime}} \hat{a}_{\kappa \uparrow}^{\dagger} \hat{a}_{-\kappa \downarrow}^{\dagger} \hat{a}_{-\kappa^{\prime} \downarrow} \hat{a}_{\kappa^{\prime} \uparrow}
$$

is the BCS type electron-electron pairing interaction term due to exchange of phonons. And,

$$
H_{3}=\sum_{l, m, \kappa} u_{l, m}^{k} \hat{a}_{\kappa \uparrow}^{\dagger} \hat{a}_{-\kappa \downarrow}^{\dagger} \hat{b}_{l \uparrow} \hat{b}_{m \uparrow}+h . c
$$

$$
\text { (h.c= hermitian conjugate) }
$$

is the new term describing the interaction between conduction and localized electrons with the coupling constant $\mathrm{u}$. the Hamiltonian in (1) will be used to determine the equations of motion in terms of the Green function.

\section{PAIRING OF SUPERCONDUCTING AND FERROMAGNETIC ORDER PARAMETERS}

The Green's function is useful because they can be used to describe the effect of retarded interactions and all quantities of physical interest can be derived from them [27]. To get the equation of motion we use the double-time temperature dependent retarded Green function is given by (Zubarev) [24]:

$$
\begin{aligned}
& G_{r}\left(t-t^{\prime}\right) \equiv \ll \hat{A}(t) ; \hat{B}\left(t^{\prime}\right) \gg \\
& \text { or } G_{r}\left(t, t^{\prime}\right)=-i \theta\left(t-t^{\prime}\right)<\left[\hat{A}(t), \hat{B}\left(t^{\prime}\right)>\right.
\end{aligned}
$$

Where $\hat{A}$ and $\hat{B}$ are Heisenberg operators and $\theta\left(t-t^{\prime}\right)$ is the Heaviside step function. Now, using Dirac delta function and Heisenberg operators, we can write as;

$$
\left.i \frac{d}{d t} G_{r}\left(t-t^{\prime}\right)=\delta\left(t-t^{\prime}\right)<\left[\hat{A}(t), \hat{B}\left(t^{\prime}\right)\right]>+<<[\hat{A}(t), H], \hat{B}\left(t^{\prime}\right)\right]>>
$$

The Fourier transformation $G_{r}(\omega)$ is given by 
Taking the Fourier transform of $(8)$, we get:

$$
G_{r}\left(t-t^{\prime}\right)=\int G_{r}(\omega) \exp \left[-i \omega\left(t-t^{\prime}\right)\right] d \omega
$$

From (10), it follows that

$$
\left.\omega G_{r}(\omega)=<\left[\hat{A}(t), \hat{B}\left(t^{\prime}\right)\right]>_{\omega}+\ll[\hat{A}(t), H], \hat{B}\left(t^{\prime}\right)\right] \gg_{\omega}
$$

$$
\omega \ll \hat{a}_{\kappa \uparrow}, \hat{a}^{\dagger}{ }_{\kappa \uparrow} \gg=1+\ll\left[\hat{a}_{\kappa \uparrow}, H\right], \hat{a}^{\dagger}{ }_{\kappa \uparrow} \gg
$$

where the anti-commutation relation,

$$
\left\{\hat{a}_{\kappa \sigma}, \hat{a}_{k^{\prime} \sigma^{\prime}}\right\}=\delta_{k k^{\prime}} \delta_{\sigma \sigma^{\prime}}
$$

has been used. To derive an expression for $\left\langle\hat{a}_{\kappa \uparrow}, \hat{a}^{\dagger}{ }_{\kappa \uparrow} \gg\right.$, we have calculate the commutator $\left[\hat{a}_{\kappa \uparrow}, H\right]$, using (2), (3), and (4). Using the identities and

$[A, B C]=\{A, B\} C-B\{A, C\}$ and $[A B, C]=A\{B, C\}-\{A, C\} B$

$$
\begin{aligned}
& {\left[\hat{a}_{\kappa \uparrow}, \widehat{H}\right]=\left[\hat{a}_{\kappa \uparrow}, \widehat{H}_{1}+\widehat{H}_{2}+\widehat{H}_{3}\right]} \\
& =\left[\hat{a}_{\kappa \uparrow}, \widehat{H}_{1}\right]+\left[\hat{a}_{\kappa \uparrow}, \widehat{H}_{2}\right]+\left[\hat{a}_{\kappa \uparrow}, \widehat{H}_{3}\right]
\end{aligned}
$$

The commutation with the interaction Hamiltonian of localized electron from the above equation of the second part as follows;

$$
\left[\hat{a}_{\kappa \uparrow}, H_{1}\right]=\epsilon_{\kappa} \hat{a}_{\kappa, \uparrow}
$$

After some lengthy but straightforward calculations; we arrive at the following results;

$$
\begin{aligned}
& {\left[\hat{a}_{\kappa \uparrow}, H_{2}\right]=-\sum_{p} V_{k p} \hat{a}_{-\kappa \downarrow}^{\dagger} \hat{a}_{-p \downarrow} \hat{a}_{p \uparrow}} \\
& {\left[\hat{a}_{\kappa \uparrow}, H_{3}\right]=\sum_{l, m} u_{l, m}^{k} \hat{a}_{-\kappa \downarrow}^{\dagger} \hat{b}_{l \uparrow} \hat{b}_{m \uparrow}}
\end{aligned}
$$

Plugging (13) in to (8), we get

$$
\begin{gathered}
\omega \ll \hat{a}_{\kappa \uparrow}, \hat{a}^{\dagger}{ }_{\kappa \uparrow} \gg=1+\epsilon_{\kappa} \ll \hat{a}_{\kappa \uparrow}, \hat{a}^{\dagger}{ }_{\kappa \uparrow} \gg-\sum_{p} V_{p k} \ll \hat{a}_{-\kappa \downarrow}^{\dagger} \hat{a}_{-p \downarrow} \hat{a}_{p \uparrow}, \hat{a}^{\dagger}{ }_{\kappa \uparrow} \gg \\
+\sum_{k} u_{l, m}^{k} \ll
\end{gathered}
$$

Using Wick's theorem for factorization ( Schwabl) [25], we can reduce (17) into the following form,

Where

$$
\left(\omega-\epsilon_{\kappa}\right) \ll \hat{a}_{\kappa \uparrow}, \hat{a}^{\dagger}{ }_{\kappa \uparrow} \gg=1-(\Delta-\gamma) \ll \hat{a}_{-\kappa \downarrow}^{\dagger}, \hat{a}_{\kappa \uparrow}^{\dagger}{ }_{\kappa \uparrow} \gg
$$

$$
\begin{aligned}
& \Delta=V \sum_{P}\left\langle\left\langle\hat{a}_{-P \downarrow}, \hat{a}_{P \uparrow} \gg=V \sum_{P}\left\langle\hat{a}_{-P \downarrow}^{\dagger}, \hat{a}_{P \uparrow}^{\dagger}\right\rangle\right.\right. \\
& \gamma=u \sum_{K}\left\langle\hat{b}_{l \uparrow}, \hat{b}_{m \uparrow}\right\rangle=u \sum_{K}\left\langle\hat{b}_{l \uparrow}^{\dagger}, b^{\dagger}{ }_{m \uparrow}\right\rangle
\end{aligned}
$$

are related to the superconducting and ferromagnetic order parameters respectively, and are assumed to be real. To determine $\ll \hat{a}_{-\kappa \downarrow}^{\dagger}, \hat{a}_{\kappa \uparrow}^{\dagger} \gg$ in (15), we use (7), to obtain

where (9) has been used,

$$
\omega \ll \hat{a}_{-\kappa \downarrow}^{\dagger}, \hat{a}^{\dagger}{ }_{\kappa \uparrow} \gg=\left\langle\left[\hat{a}_{-\kappa \downarrow}^{\dagger}, H\right], \hat{a}_{\kappa \uparrow}^{\dagger} \gg\right.
$$

Proceeding in the same manner, we can reduce (18) into the form:

$$
\left.\left(\omega+\epsilon_{\kappa}\right) \ll \hat{a}_{-\kappa \downarrow}^{\dagger}, \hat{a}_{\kappa \uparrow}^{\dagger}\right\rangle=-(\Delta-\gamma) \ll \hat{a}_{\kappa \uparrow}, \hat{a}_{\kappa \uparrow}^{\dagger} \gg
$$

Eliminating $\ll \hat{a}_{\kappa \uparrow}, \hat{a}_{\kappa \uparrow}^{\dagger} \gg$ from (18) and (22), we obtain,

$$
\ll \hat{a}_{-\kappa \uparrow}^{\dagger}, \hat{a}^{\dagger}{ }_{\kappa \uparrow} \gg=\frac{-(\Delta-\gamma)}{\left(\omega^{2}-\epsilon_{\kappa}^{2}-(\Delta-\gamma)^{2}\right)}
$$

To take into account the temperature dependence of order parameters, we shall rewrite (16) and (17) as:

Where $\beta=\frac{1}{K T}$

$$
\begin{aligned}
& \Delta=\frac{V}{\beta} \sum_{\kappa} \ll \hat{a}_{-\kappa \downarrow}^{\dagger}, \hat{a}_{\kappa \uparrow}^{\dagger} \gg \\
& \gamma=\frac{u}{\beta} \sum_{k} \ll \hat{b}_{l \uparrow}^{\dagger}, b^{\dagger}{ }_{m \uparrow} \gg
\end{aligned}
$$

Doing a lot as [17], we arrived at the following results: 


$$
\frac{\Delta}{\alpha}=\int_{0}^{\hbar \omega_{b}} \frac{(\Delta-\gamma)}{\sqrt{\left(\epsilon^{2}+(\Delta-\gamma)^{2}\right)}} \tanh \left(\beta \sqrt{\epsilon^{2}+(\Delta-\gamma)^{2}} / 2\right) d \epsilon
$$

$\alpha=N(0) V$, Where $N(0)$, is the density of states at the Fermi level.

From (23), it clearly follows that the order parameters $\Delta$ and $\gamma$, for superconductivity and magnetism are interdependent.

And

Where

$$
\ll \hat{b}_{m \uparrow}^{\dagger}, \hat{b}_{l \uparrow}^{\dagger} \gg=\frac{\Delta_{l m}}{\left(\omega^{2}-\epsilon_{l}^{2}-\Delta_{l m}^{2}\right)}
$$

$$
\begin{gathered}
\Delta_{l m}=\sum_{k} u_{k}^{l m}\left\langle\hat{a}_{k \uparrow}^{\dagger}, \hat{a}_{-k \downarrow}^{\dagger} \gg\right. \\
\frac{\gamma}{\alpha}=\Delta_{l m} \int_{0}^{\hbar \omega_{b}} \frac{1}{\sqrt{\left(\epsilon^{2}+\Delta_{l m}^{2}\right.}} \tanh \left(\beta \sqrt{\epsilon^{2}+\Delta_{l m}^{2}} / 2\right) d \epsilon
\end{gathered}
$$

From (25), it is again evident that the order parameters $\Delta$ and $\gamma$ are interdependent, as was the case from (23).

It is, therefore, possible that in some temperature interval, ferromagnetism and superconductivity can coexist, although one phase has a tendency to suppress the critical temperature and the order parameter of the other phase.

\section{DEPENDENCE OF THE MAGNETIC ORDER PARAMETER ON THE TRANSITION TEMPERATURE FOR SUPERCONDUCTIVITY AND FERROMAGNETISM}

To study how $\gamma$ depends on the superconducting transition temperature $\mathrm{T}_{\mathrm{C}}$, we consider the case, when $T \rightarrow 0 K, \beta \rightarrow \infty$

We can then replace

in (23) and get,

$$
\tanh \left(\beta \sqrt{\epsilon^{2}+(\Delta-\gamma)^{2}} / 2\right) \rightarrow 1
$$

$$
\begin{array}{r}
\frac{\Delta}{\alpha}=\int_{0}^{\hbar \omega_{b}} \frac{(\Delta-\gamma)}{\sqrt{\left(\epsilon^{2}+(\Delta-\gamma)^{2}\right)}} d \epsilon \\
\frac{1}{\alpha}=\left(1-\frac{\gamma}{\Delta}\right) \sinh ^{-1}\left(\frac{\hbar \omega_{b}}{\Delta-\gamma}\right)
\end{array}
$$

since $\left(\frac{\hbar \omega_{b}}{\Delta-\gamma}\right)^{2} \gg 1$ the above equation become to:$$
\Delta-\gamma=2 \hbar \omega_{b} \exp \left(-\frac{1}{\alpha\left(1-\frac{\gamma}{\alpha}\right)}\right)
$$

from the BCS theory, the order parameter $\Delta$, at $\mathrm{T}=0$ for a given superconductor with transition temperature $\mathrm{T}_{\mathrm{C}}$ is given by

using this result in (27), we obtain

$$
2 \Delta(0)=3.53 k_{B} T_{c}
$$

$$
\gamma=1.75 k_{B} T_{c}-2 \hbar \omega_{b} \exp \left(-\frac{1}{\alpha\left(1-\frac{\gamma}{1.75 k_{B} T_{c}}\right)}\right)
$$

Once we know $\alpha$, we can solve (29) numerically to draw the phase diagram for $\gamma$ and $\mathrm{T}_{\mathrm{C}}$.

To estimate $\alpha$, we consider the case,

From (23), we then have

$$
\text { which implies, } \Delta \rightarrow 0 \text { T } T \rightarrow T_{c}
$$




$$
\begin{gathered}
\begin{array}{c}
\frac{1}{\alpha}=\int_{0}^{\hbar \omega_{b}} \frac{1}{\sqrt{\left(\epsilon^{2}+\gamma^{2}\right)}} \tanh \left(\beta \sqrt{\epsilon^{2}+\gamma^{2}} / 2\right) d \epsilon \\
\quad-\lim _{\Delta \rightarrow 0} \int_{0}^{\hbar \omega_{b}} \frac{\gamma}{\Delta \sqrt{\left(\epsilon^{2}+(\Delta-\gamma)^{2}\right)}} \tanh \left(\beta \sqrt{\epsilon^{2}+(\Delta-\gamma)^{2}} / 2\right) d \epsilon \\
\frac{1}{\alpha}=I_{1}-I_{2}
\end{array} \\
\text { Putting } E^{2}=\epsilon^{2}+\gamma^{2}
\end{gathered}
$$

we can write

Using the result

$$
I_{1}=\int_{0}^{\hbar \omega_{b}} \frac{1}{E} \tanh (\beta E / 2) d \epsilon
$$

$$
I_{1}=\int_{0}^{x} \frac{\tanh x}{x} d x=\left.(\ln x)(\tanh x)\right|_{0} ^{x}-\int_{0}^{x} \frac{\ln x}{\cosh ^{2} x} d x
$$

and the fact that, for low temperature, $\tanh \left(\frac{\hbar \omega_{b}}{2 k_{B} T}\right) \rightarrow 1$,

we have from (31),

$$
I_{1}=\ln \left(\frac{\hbar \omega_{b}}{2 k_{B} T_{c}}\right)-\ln \left(\frac{\pi}{4 \delta}\right)
$$

Where $\gamma$ is the Euler constant having the value $\gamma=1.78$ (Hsian) [28] we can write (32) as,

$$
I_{1}=\ln \left(1.14 \frac{\hbar \omega_{b}}{k_{B} T_{c}}\right)
$$

Using L' Hospital's rule, it is easy to show that

which can be neglected since $\gamma^{2}$ is very small.

$$
I_{2}=-\int_{0}^{\hbar \omega_{b}}\left(\gamma^{2} \beta\right) \frac{\operatorname{sech}^{2}\left(\frac{\beta \sqrt{\epsilon^{2}+\gamma^{2}}}{2}\right)}{2\left(\epsilon^{2}+\gamma^{2}\right)} d \epsilon
$$

Substituting (33) in (30), we then obtain

This implies,

$$
\frac{1}{\alpha}=\ln \left(1.14 \frac{\hbar \omega_{b}}{k_{B} T_{c}}\right)
$$

$$
T_{c}=\frac{1.14 \hbar \omega_{b}}{k_{B}} \exp \left(-\frac{1}{\alpha}\right),
$$

which can be used to estimate $\exp \left(-\frac{1}{\alpha}\right)$ for UCoGe, UIr \& $\mathrm{UGe}_{2}$, using the experimental value $T_{c} \approx 0.7 k$, $T_{c} \approx 0.18 \mathrm{k}$ and $T_{c} \approx 0.8 \mathrm{k}$ for these compounds.

To study how $\gamma$ depends on the magnetic transition temperature Tm, we consider (25). Neglecting $\Delta^{2}$, and proceeding as before, it is easy to show that,

This gives;

$$
\gamma \approx-(\alpha \Delta) \ln \left(1.14 \frac{\hbar \omega_{b}}{k_{B} T_{m}}\right)
$$

$$
\therefore T_{m}=\left(\frac{1.14 \hbar \omega_{b}}{k_{B}}\right) \exp \left(\frac{\gamma}{\alpha \Delta}\right)
$$

From (28), we can estimate as $\Delta(0) \approx 1.706 \times 10^{-23} \mathrm{~J}, \Delta(0) \approx 0.439 \times 10^{-23} \mathrm{~J}$ and $\Delta(0) \approx 1.949 \times 10^{-23} \mathrm{~J}$ for $\mathrm{UCoGe}$, UIr \& $\mathrm{UGe}_{2}$ respectively, using the known value of $\mathrm{T}_{\mathrm{C}}$. So,

we can use (36) to draw the phase diagram for $\gamma$ and Tm

\section{COUPLING OF TRIPLET SUPERCONDUCTIVITY AND FERROMAGNETISM}

Superconductivity in these systems is supposed to be triplet regardless of the mechanism of pairing which could be due to some unspecified mechanism possibly due to spin fluctuations near the quantum critical point as in He3. These allow fermions to condense into cooper pairs with $\ell=1,3, \ldots$ states rather than into $\ell=0,2, \ldots, s$ and states of BCS theory [28]. Following [29], the gap function or parameter can be articulated as: 


$$
\Delta_{k \sigma \sigma^{\prime}}=-\sum_{k} V_{\sigma \sigma^{\prime} \alpha \beta}\left(k, k^{\prime}\right) \ll a_{k^{\prime} \alpha} a_{-k^{\prime} \beta} \gg
$$

The correlation $\left\langle a_{k^{\prime} \alpha} a_{-k^{\prime} \beta} \gg\right.$ can be intended as [30]:

$$
F(k, \beta)=\frac{1}{2 E_{k \uparrow}}\left[\frac{d(k)+q(k) x d(k)}{|q(k)|}\right] \tanh \left(\frac{\beta}{2}\right) E_{k \uparrow}+\frac{1}{2 E_{k \downarrow}}\left[\frac{d(k)-q(k) x d(k)}{|q(k)|}\right] \tanh \left(\frac{\beta}{2}\right) E_{k \downarrow}
$$

Where $E_{k \uparrow, \downarrow}=\left[E_{k}^{2}+\left|d(k)^{2}\right| \pm q(k)\right]^{1 / 2}$

For the triplet state, we have to write as:

$$
\Delta(k) \Delta^{*}(k)=\left|d(k)^{2}\right| \hat{I}+i \sigma q(k)
$$

At this point, the triplet states are $|\downarrow \downarrow\rangle,|\uparrow \downarrow+\downarrow \uparrow\rangle$ and $|\uparrow \uparrow\rangle$.

For unitary pairing states, $\mathrm{q}(\mathrm{k})=0$ for all $\mathrm{k}, F(\mathrm{k}, \beta)$ becomes

$$
F(k, \beta)=\frac{\Delta(k)}{E_{k}} \tanh \left(\frac{\beta}{2}\right) E_{k}
$$

which leads to s-wave superconductivity expression for $\mathrm{T}_{\mathrm{C}}$ known by

$$
T_{c}=\frac{1.14 \hbar \omega_{b}}{k_{B}} \exp \left(-\frac{1}{\alpha}\right)
$$

This will be true for opposite spin states as singlet states are also opposite spin states. For triplet states, the gap function can be written as [30]:

$$
\Delta_{k \sigma \sigma^{\prime}}=\sum_{k} V\left(k, k^{\prime}\right)\left\{\frac{1}{2 E_{k \uparrow}}\left[d(k)+\frac{q(k) x d(k)}{|q(k)|^{2}}\right] \tanh \left(\frac{\beta}{2}\right) E_{k \uparrow}+\frac{1}{2 E_{k \downarrow}}\left[d(k)-\frac{q(k) x d(k)}{|q(k)|^{2}}\right] \tanh \left(\frac{\beta}{2}\right) E_{k \downarrow}\right\}
$$

This expression can be recast into four equal spin states with $\mathrm{q}(\mathrm{k})=0$ for all $\mathrm{k}$

$$
1=\sum N(\epsilon) V \frac{\left[\tanh \left(\frac{\beta c}{2}\right)\left(E_{k}-\sigma \mu B . H\right)\right]}{E_{k}-\sigma \mu B . H}
$$

With $E_{K \uparrow}=E_{K \downarrow}=E_{K}$. Here, we have introduced energy dependent density of states with $\sigma= \pm$ for $\uparrow$ or $\downarrow$ spins. By changing summation into integral, we can get:

$$
k_{B} T_{C}=1.14 \hbar \omega_{b} \exp \left\{\left(-V\left[\left.N\left(\epsilon_{\ell}\right)\right|_{\epsilon_{f}} \pm\left.\frac{\partial N(\epsilon)}{\partial \epsilon}\right|_{\epsilon_{f}} \sigma \mu_{B} H\right]\right)^{-1}\right\}
$$

we can rewrite this as:

$$
k_{B} T_{C}=1.14 \hbar \omega_{b} \exp \left(-\frac{1}{\alpha \pm \alpha^{\prime}}\right)
$$

Where $\alpha=\left.V N\left(\epsilon_{\ell}\right)\right|_{\epsilon_{f}}$ and $\alpha^{\prime}=\sigma V a \mu_{B} H, \quad a=\left.\frac{\partial N(\epsilon)}{\partial \epsilon}\right|_{\epsilon_{f}}$.

Here, $H$ can be an applied field or exchange magnetic field.

From this, we can write

$$
\begin{aligned}
& k_{B} T_{C \uparrow}=1.14 \hbar \omega_{b} \exp \left(-\frac{1}{\alpha+\alpha^{\prime}}\right) \\
& k_{B} T_{C \downarrow}=1.14 \hbar \omega_{b} \exp \left(-\frac{1}{\alpha-\alpha^{\prime}}\right)
\end{aligned}
$$

From the above two expression we can generalized $T_{C \uparrow}$ for up spin fermions will be larger than $T_{C \downarrow}$ for down ones. Therefore, in between $T_{C \uparrow}$ and $T_{C \downarrow}$, there is no pairing. It appears that because of the exchange coupled uranium $f$ electron spins $|\downarrow \downarrow\rangle$ and $|\uparrow \downarrow+\downarrow \uparrow\rangle$ of the triplet set may be make disappeared or suppressed giving principally $|\uparrow \uparrow\rangle$. This may be the limitation of the model. 


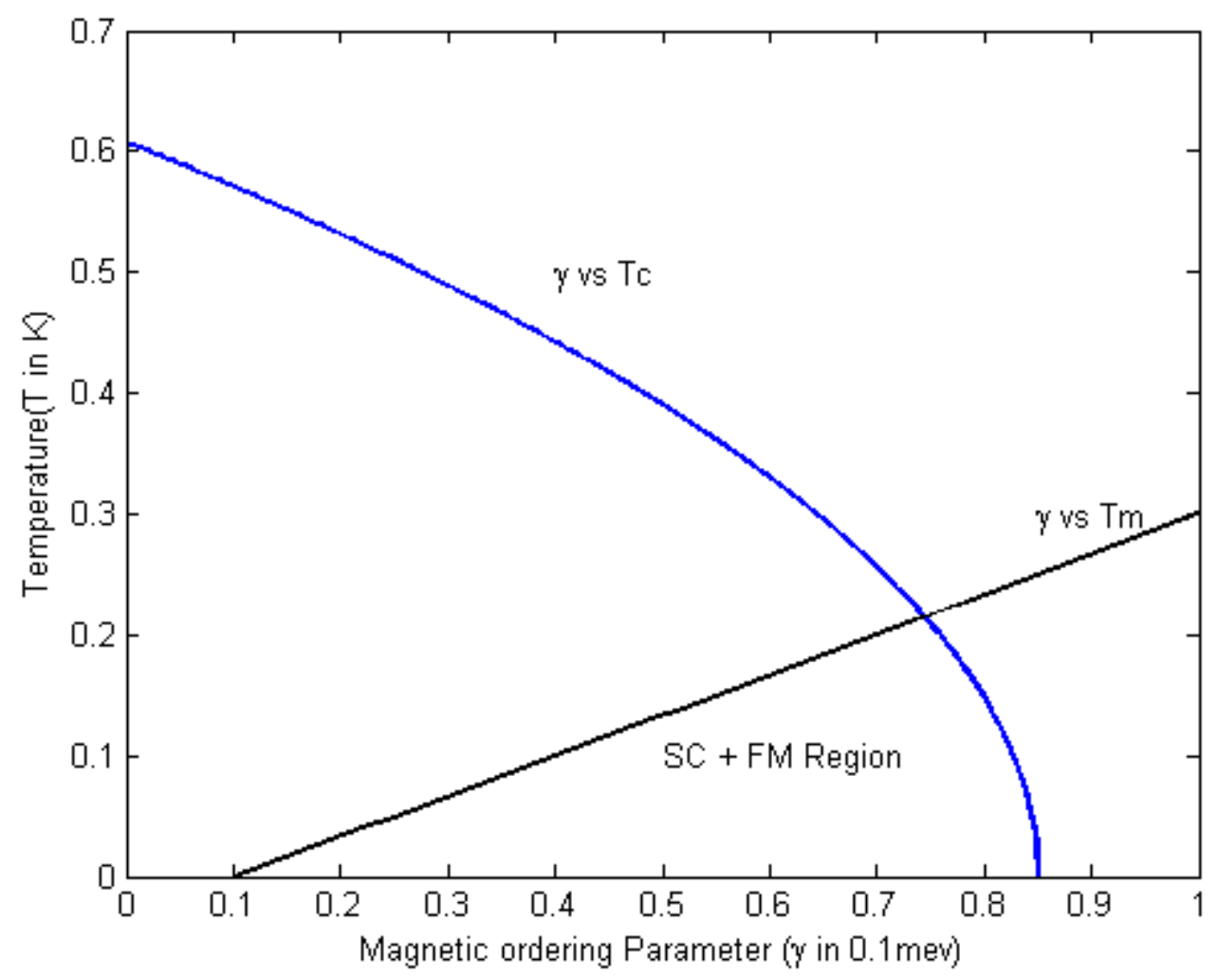

Fig. 1: Co-existence of superconductivity and ferromagnetism in UCoGe.

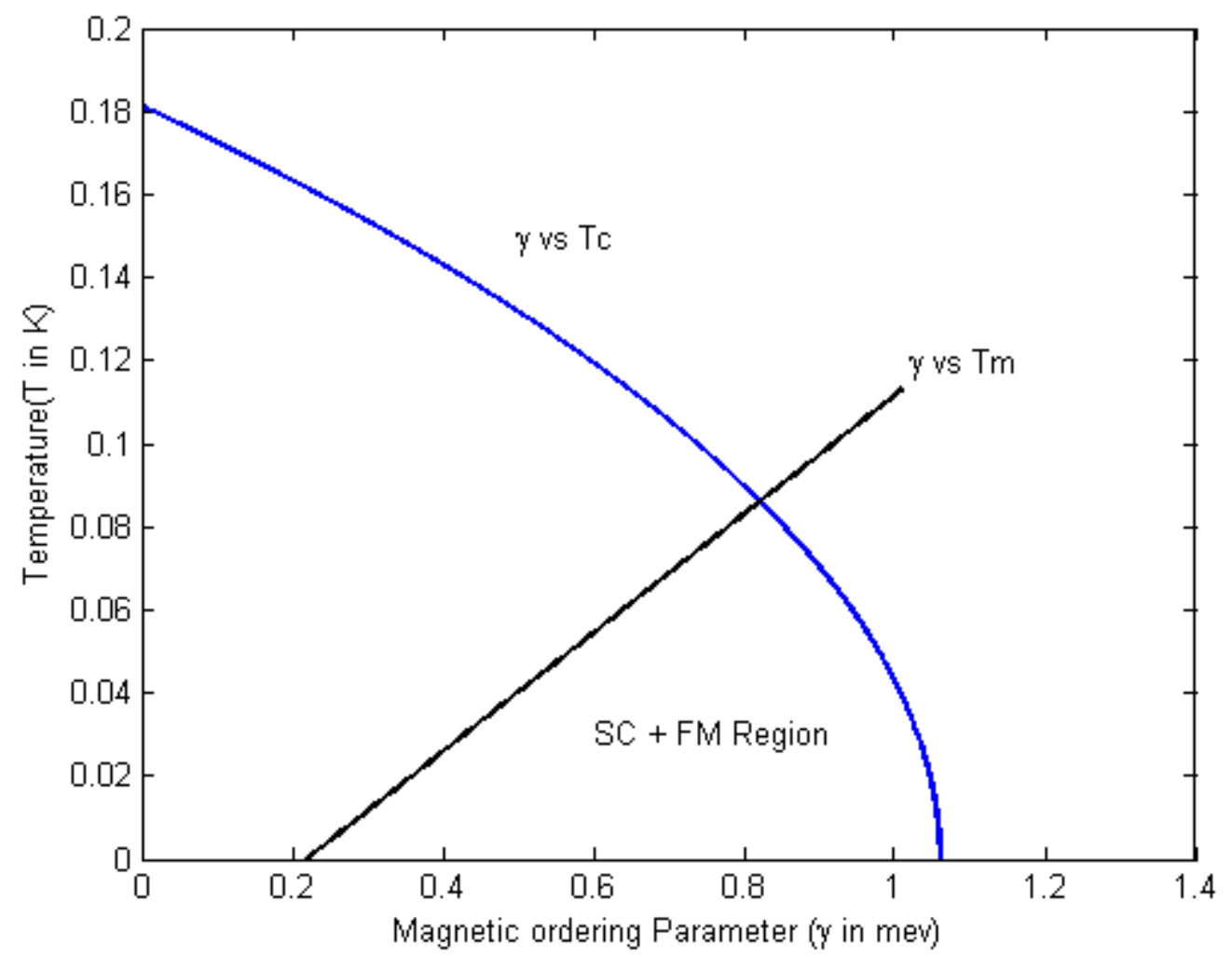

Fig. 2: Co-existence of superconductivity and ferromagnetism in UIr. 


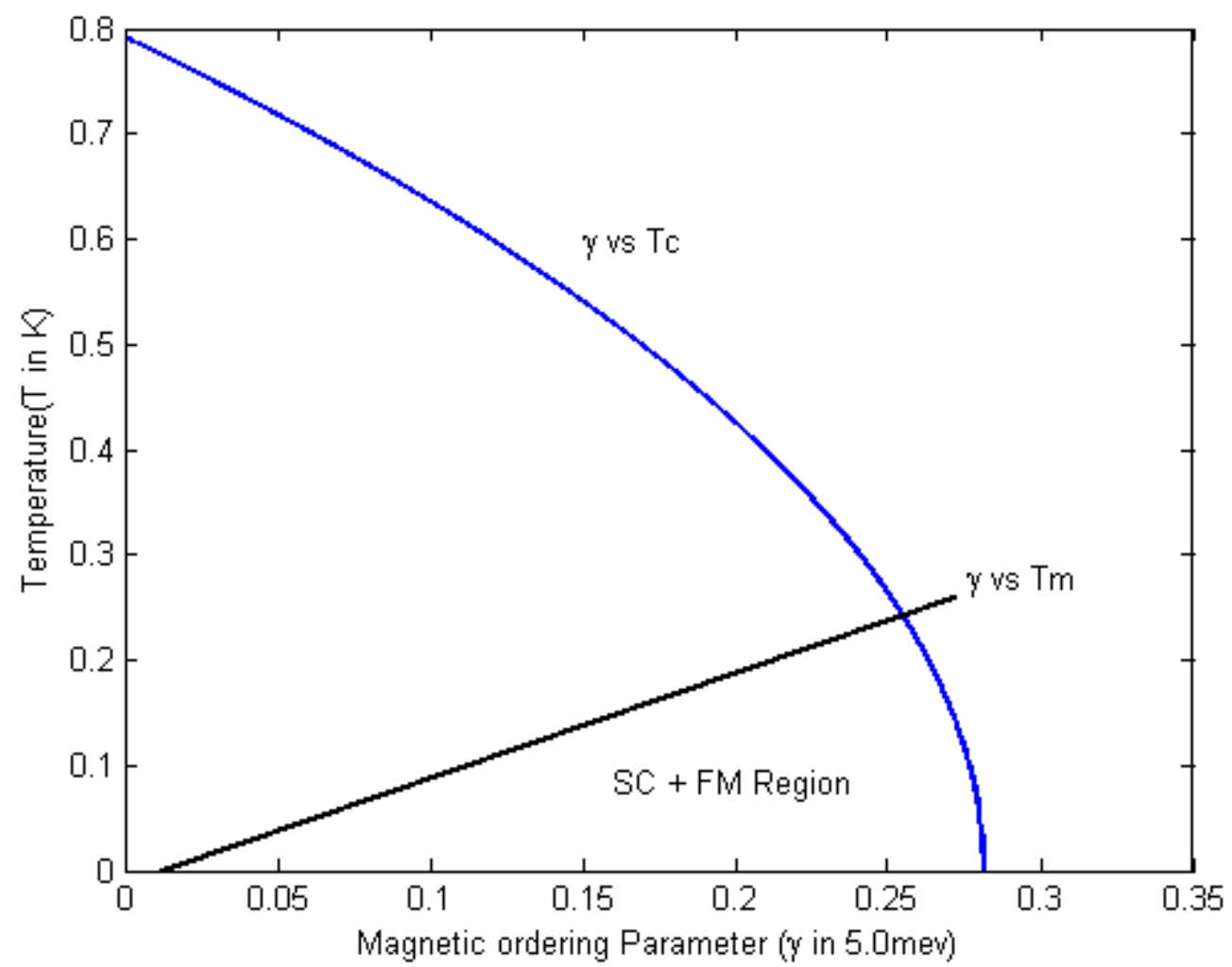

Fig. 3: Co-existence of superconductivity and ferromagnetism in $\mathrm{UGe}_{2}$.

\section{RESULTS AND CONCLUSION}

In uranium intermetallic compounds magnetism has a strong itinerant character and both ordering phenomena are carried by 5f electrons .In Fig. 1, 2 and 3 we have presented the theoretical curve of the magnetic order parameter $\gamma$ as a function of the superconducting temperature $\mathrm{T}_{\mathrm{C}}$. For this purpose, we have used (29) which has been numerically solved using the relevant parameters for UCoGe, UIr and $\mathrm{UGe}_{2}$. In the same figure, we have also plotted the curve of $\gamma$ as a function of the magnetic transition temperature $T_{m}$, using (36). This curve is found to be almost linear up to the experimental value of $T_{m}=3 \mathrm{k}, T \mathrm{~m}=46 \mathrm{k}$ and $T \mathrm{~m}=52 \mathrm{k}$ for Uranium-Based Superconductors of UCoGe, UIr and $\mathrm{UGe}_{2}$ that we study.

From Fig. 1, 2 and 3 we observe that $\mathrm{T}_{\mathrm{C}}$ decreases with increase in $\gamma$, whereas $\mathrm{Tm}$ increases with increase in $\gamma$. The superconducting and ferromagnetic phases, therefore, resist each other. However, the present work shows that there is a small region of temperature, where both the phases may be in existence together and indicated by $(\mathrm{SC}+\mathrm{FM})$ in the Figures. Thus a simple model based on a Hamiltonian which takes into account the spin interactions between conduction electrons and ferromagnetically ordered localized electrons, can explain the co-existence of singlet superconductivity and ferromagnetism in UCoGe, UIr and $\mathrm{UGe}_{2}$. In the triplet case, it appears the state $|\uparrow \uparrow\rangle$ will have preponderance and other states like $|\downarrow \downarrow\rangle$ and $|\uparrow \downarrow+\downarrow \uparrow\rangle$ will be suppressed with small magnetic field applied or resulting from exchange interaction. $T_{c \uparrow}$ is found to be larger than $T_{c \downarrow}$. Thus, only spin up electrons will pair and compress first. Our study unequivocally shows that superconductivity and itinerant ferromagnetism truly coexist in these systems and both ordering phenomena are carried by the same $5 f$ electrons.

\section{ACKNOWLEDGEMENTS}

I thank Prof. Amarendra Rajput for providing me constructive comments, suggestions and valuable support.

\section{REFERENCES}

[1] S.S. Saxena., et al.: Nature 406, 2000, 587.

[2] S.K. Sinha, , G.W. Crabtree, D.G. Hinks, H.A. Mook and O.A. Pringle, Neutron scattering studies of magnetic superconductors. J.Magn and Magn.mater, 1983, 31-34 part, 2: 489.

[3] J.W. Lynn, A. Raggazoni, R. Pynn and J. Joffrin, Observation of long range magnetic order in the reentrant superconductor HoMo6S8. J.Physique Lett, 1981, 42: L- 45-L-49.

[4] J.L. Genicon, , J.P. Modon Danon., R.Tournier, O. Peña, R. Horyn, and M. Sergent, Percolation, 1984. 
[5] N.T. Huy, A. Gasparini, D.E. de Nijs, Y. Huang, J.C.P. Klaasse, T. Gortenmulder, A. de Visser, A. Hamann, T. Görlach and H. v. Löhneysen, Phys. Rev. Lett, 99, 2007,067006.

[6] A. de Visser, in: Encyclopedia of Materials: Science and Technology, eds K.H.J. Buschow, R.W. Cahn, M.C. Flemings, B. Ilschner, E.J. Kramer, S. Mahajan and P. Veyssiere (Elsevier, Oxford, 2010), pp. 1-6.

[7] S.S. Saxena, K. Ahilan, P. Agarwal, F.M. Grosche, R.K. Haselwimmer, M. Steiner, E. Pugh, I.R. Walker, S.R. Julian, P. Monthoux, G.G. Lonzarich, A.D. Huxley, I. Sheikin, D. Braithwaite and J. Flouquet, Nature (London) 406, 2000, 587.

[8] D. Aoki, A.D. Huxley, E. Ressouche, D. Braithwaite, J. Flouquet, J.P. Brison, E. Lhotel and C. Paulsen, Nature (London) 413, 2001, 613.

[9] T. Akazawa, H. Hidaka, T. Fujiwara, T.C. Kobayashi, E. Yamamoto, Y. Haga, R. Settai and Y. Onuki, J. Phys.: Condens. Matter 16, 2004, L29.

[10] N.F. Berk and J.R. Schrieffer, Phys. Rev. Lett. 17, 1966, 433.

[11] A. Kolodzieyczyk, B.V.B. Sarkissian, B.R. Coles, J. Phys. F. Met. Phys, 10, 1980, L333.

[12] S.S. Saxena, , P. Agarwal, K. Ahilan, F.M. Grosche, R.K.W. Hasselwimmer, M.J. Steiner, E. Pugh, I.R. Walker, S.R. Julian, P. Monthoux, G.G. Lonzarich, A. Huley, I. Sheikin, D. Braithwaite, J. Flouquet, Nature 406, 2001, 613.

[13] C. Pfleiderer, M. Uhlarz, S.M. Hayden, R. Vollmer, R. Von Lohneysen, N.R. Bernhoeft, G.G. Lonzarich, Nature 412, 2001, 58.

[14] N.T. Huy, A. Gasparini, D.E. de Nigs, Y. Huang, J.C.P. Klaasse, T. Gortenmulder, A. de Visser, A. Hamann, T. Gorlach, H.V. Lohneyser, Phys. Rev. Lett. 99, 067006 (2007).

[15] K.H.J. Buschow, E. Brück, R.G. van Wierst, F.R. de Boer, L. Havela, V. Sechovský, P. Nozar, E. Sugiura, M. Ono, M. Date and A. Yamagishi, J. Appl. Phys. 67, 1990, 5215.

[16] N.T. Huy and A. de Visser, Solid State Comm. 149, 2009, 703.

[17] H. Brhane, A. Rajput, G. Abeb,. Coexistence of Superconductivity and Ferromagnetism in a Magnetic Superconductor. The IJES. Vol. 2(8) 2013, 17-23.

[18] A.J. Legget, Rev. Mod. Phys. 47, 1975, 331.

[19] D. Fay, J. Appel, Phys. Rev. B 22, 1980, 3173.

[20] M. Ishikawa, et al.., J. Solid State Commun, 23, 1977, 37.

[21] Fertig, W.A.: Phys. Rev. Lett. 38, 377 (1977).

[22] N.I. Karchev, Blagoev, K.B., Bedell, K.S., Littlewood, P.B.: Phys.Rev. Lett. 86, 2001, 846.

[23] S. Debaand, Phys. Rev. B 66, 140505(R) (2002).

[24] D. N. Zubarev, Double-time green functions in statistical physics.usp. Fiz. Nauk. Sssr 71:7; Translation: Sov. Phys. Usp. 3, 1960, 320-345.

[25] F. Schwabl, Advanced Quantum Mechanics. Springer-Verlag Berlin Heidelberg, 2009.

[26] P. C. Hsian, Robust based band reed solomon detection over Power line channel. J.of Engg. Sc. and Tech; 6(1), 2011, $69-81$.

[27] G. Rickayzen, "Theory of superconductivity", John Wiley and Sons. Inc. New York (Interscience Publishers): 1965, 483p.

[28] A.J. Legget, Rev. Mod. Phys. 47, 1975, 331.

[29] M. Sigrist, Ueda, K.: Rev. Mod. Phys. 63, 1990, 239.

[30] Subhra Kakani, Coexistence of superconductivity and Itinerant ferromagnetism in UCoGe. IJRAP. VGol.3(1) 2014, 39-59. 